\title{
Factores pronósticos en la recidiva y progresión del cáncer superficial vesical. Grupos de riesgo (Parte II)
}

\author{
García Rodríguez J, Fernández Gómez JMª, Escaf Barmadah S, Jalón Monzón A, \\ Álvarez Múgica M, Regadera Sejas J.
}

\begin{abstract}
Servicio de Urología I. Hospital Central de Asturias. Departamento de Especialidades Médico-Quirúrgicas. Facultad de Medicina. Universidad de Oviedo. Oviedo.
\end{abstract}

Actas Urol Esp. 2006;30(10):1009-1016

\section{RESUMEN}

FACTORES PRONÓSTICOS EN LA RECIDIVA Y PROGRESIÓN DEL CÁNCER SUPERFICIAL VESICAL. GRUPOS DE RIESGO (PARTE II)

Introducción: Intentamos establecer grupos de riesgo en función de la combinación de las características de cada tumor vesical, para un mejor seguimiento de estos pacientes.

Material y Métodos: Una vez conocidas las variables implicadas en recurrencia y progresión analizamos los datos para establecer los grupos extremos, es decir, los de peor y mejor pronóstico, quedando un grupo residual que correspondería al de los tumores de pronóstico intermedio.

Resultados: A partir de los resultados obtenidos, nos hemos planteado establecer tres grupos de riesgo: Grupo de mal pronóstico (alto riesgo): Tumores de alto grado (G3), Tis aislado o asociado y tumores de grado 2 múltiples o recurrentes que hubieran recidivado en menos de 6 meses; Grupo de pronóstico intermedio: Tumores TaG1 múltiples, así como neoplasias Ta-1G2 y T1G1 no múltiples (menos de 3 tumores) y Grupo de bajo riesgo: Tumores TaG1 únicos.

Discusión: De acuerdo con estos resultados el grupo de mal pronóstico, conlleva un alto riesgo de recidiva y progresión. El tratamiento más efectivo resultó la BCG (bacilo Calmette-Guerin) con mantenimiento, exigiendo, un seguimiento prolongado, más intenso en los 2 primeros años tras la RTU. En el grupo de pronóstico intermedio, las tasas de recidiva superficial a largo plazo fueron tan altas como las de alto riesgo, requiriéndose un seguimiento largo, en éste resultó efectivo el empleo de quimioterapia o BCG siendo preferible el mantenimiento. Por último, en el grupo de buen pronóstico no objetivamos progresiones, siendo el tiempo hasta la recidiva el más largo, con estabilización de la tasa de recurrencias tras 1 año.

Palabras clave: Cáncer vesical. Superficial. Grupos de riesgo.

\section{ABSTRACT \\ PRONOSTIC FACTORS ON RECURRENCE AND PROGRESSION OF SUPERFICIAL BLADDER CANCER. RISK GROUPS (PART II)}

Introduction: We try to establish risk groups combining the characteristics of each bladder tumour, for a better monitoring of these patients.

Material and Methods: Once known the variables implied in recurrence and progression we analyze the data to establish the extreme groups, so, the one with the worse and the one with the better prognostic, remaining a residual group that would correspond of the tumours of intermediate prognostic.

Results: From the results obtained, we can establish three risk groups: Bad prognostic group (high risk) : High grade tumours (G3), isolated or associated Tis and multiple or recurrent grade 2 tumours that were recurred in less than 6 months; Intermediate prognostic group: multiple TaG1 tumours, as well as not multiple Ta-1G2 and T1G1 (less than 3 tumours) and Group of low risk: Single TaG1 tumours.

Discussion: According to these results the group of badly risk, involves a high risk of recurrence and progression. The most effective treatment was the BCG (bacillus Calmette-Guerin) with maintenance, requiring a long-term control, more intense in the 2 first years after the RTU. In the group of intermediate risk, the rates of long-term superficial recurrence were as high as those of the high risk group, being needed a long-term control, in this group was effective the treatment with chemotherapy or BCG being preferably the maintenance. Finally in the group of good risk we don't objectify progressions being the time to the recurrence the longest one, with stabilization of the rate of recurrences after one year.

Keywords: Bladder cancer. Superficial. Risk groups. 
$\mathrm{E}$ cáncer vesical ocupa la duodécima posición en frecuencia entre las neoplasias malignas en el mundo y representa la segunda localización más frecuente de los cánceres del tracto urinario en varones en España. Aunque puede aparecer en cualquier momento de la vida, la edad media de aparición es de 69 años en hombres y 71 en mujeres, con un incremento en la incidencia y en mortalidad directamente relacionado con la edad $^{1}$. La tasa de mortalidad relacionada con la enfermedad para hombres y mujeres de 65 a 69 años es de 14 y 18\% respectivamente, mientras que de 80 a 84 años es de 30 y $37 \% \%^{2}$.

En cuanto a su etiología, desarrollo y progresión, se atribuye a un origen multifactorial. Sabemos que después de una resección transuretral (RTU) completa más del 70\% de pacientes presenta una recurrencia y un 2 a $45 \%$, según los factores pronósticos, podrán mostrar progresión con invasión en profundidad de la pared vesical ${ }^{3}$.

El conocimiento de factores pronósticos fiables del tumor vesical superficial es imprescindible para predecir la evolución futura en cuanto a recurrencia y progresión de la neoplasia y, por lo tanto, suponen los pilares sobre los que se pueden plantear las distintas estrategias de seguimiento y de tratamiento. En distintos estudios se han analizado los factores implicados en la recurrencia y progresión ${ }^{3-7}$ a partir de estos se han desarrollado algunos trabajos resultantes de exhaustivas investigaciones de datos en series apropiadas y con seguimientos prolongados, que se centran en la obtención de una guía de tratamiento. En la poca bibliografía existente al respecto, se enfatiza la necesidad de que se busquen protocolos fáciles de aplicar, basados en índices y fundamentalmente en grupos de riesgo simples, que permitan su aplicación rutinaria en pacientes con carcinoma superficial de vejiga ${ }^{8-11}$.

Nos hemos planteado el estudio de una amplia población de pacientes con carcinoma vesical superficial valorando los distintos factores pronósticos que pueden estar implicados en la recurrencia y progresión. De este modo, se pretendió establecer grupos de riesgo en función de la combinación de las características de cada tumor, para un mejor seguimiento de estos pacientes, lo que nos permitirá escoger la pauta de control más adecuada.

\section{MATERIAL Y MÉTODOS}

Una vez obtenidos los resultados de variables implicadas en recurrencia y progresión en nuestra serie de 419 tumores vesicales superficiales, consideramos las que fueron significativas en el análisis univariante como covariantes en la prueba del modelo de riesgos proporcionales de Cox, con la intención de determinar el efecto conjunto de los factores pronósticos que resultan significativos de forma independiente y cuantificar los riesgos relativos ("odds ratio") e intervalos de confianza (IC) del 95\% de recurrencia y progresión (Tabla 1). Se eligió el procedimiento por pasos hacia delante, determinando el estadístico de Wald, que introduce y selecciona variables en un orden que depende del porcentaje de la varianza.

Para establecer los grupos pronósticos a partir de los factores pronósticos que resultaron independientes en el modelo de Cox, se estudiaron inicialmente los factores de mayor peso en el parámetro que representa peor evolución, en nuestro caso la progresión a tumores invasores del detrusor. A partir de aquí, analizamos los datos para establecer los grupos extremos, es decir, los de peor y mejor pronóstico, quedando un grupo residual que correspondería al de los tumores de pronóstico intermedio. Para ello, con la intención de dejar escapar el menor número de pacientes de mal pronóstico de recurrencia o progresión se

Tabla 1

Variables independientes para la recurrencia (a) y progresión (b) en el modelo de riesgo proporcional de Cox, con los odds ratio correspondientes

\begin{tabular}{lc}
\hline Recurrencia (a) & (OR) \\
\hline Inducción & 1,623 \\
Sin lavados & 2,218 \\
Dosis altas de BCG & 0,4694 \\
Técnica quirúrgica & 1,507 \\
Multiplicidad & 2,160 \\
Meses de recurrencia previa & 1,505 \\
\hline Progresión (b) & (OR) \\
\hline Inducción & 1,623 \\
Sin lavados & 2,218 \\
Dosis altas de BCG & 0,4694 \\
Técnica quirúrgica & 1,507 \\
Multiplicidad & 2,160 \\
Meses de recurrencia previa & 1,505 \\
\hline
\end{tabular}


buscó inicialmente un grupo amplio de alto riesgo de recurrencia y progresión. Además, se investigó un grupo pequeño en el que la recurrencia fuera mínima y en el que estuviéramos seguros de que no se iba a producir progresión, el cual constituiría el grupo de buen pronóstico.

Finalmente, se estudió el comportamiento de los tumores según los diferentes grupos establecidos, en relación al tiempo libre de enfermedad ( $\sin$ recidiva ni progresión) y la influencia de la terapia endovesical en la evolución de dichas neoplasias.

\section{RESULTADOS}

Para configurar los grupos de riesgo nos hemos basado en al análisis estadístico según la odds ratio (OR). Observamos, analizando las variables independientes para la recurrencia y progresión según el modelo de riesgo proporcional de Cox y las ordenamos según los OR. Así dado que, para recurrencia, el uso de dosis altas de BCG disminuía el riesgo de recurrencia con una OR<de 1, lo etiquetamos como un factor protector, mientras la multiplicidad, con una OR de 2,16 y la ausencia de terapia endovesical (OR de 2,218 ), fueron los factores que más aumentaron el riesgo de recurrencia. En cuanto a progresión el Grado III con una OR de 20,470 resulta un factor claramente agresivo.

Para establecer los grupos pronósticos a partir de los datos recogidos en nuestra serie, empezamos por estudiar los factores de mayor peso en el parámetro que representa peor evolución que, evidentemente, es la progresión con invasión detrusoriana. Analizamos los datos para establecer los grupos extremos inicialmente, es decir, los de peor y mejor pronóstico. Por ello, en principio se escogió el grado de malignidad como patrón de referencia, dado el elevado riesgo relativo de los tumores de grado 3 y Tis.

Puesto que los carcinomas de grado 2 también presentaron mayor riesgo relativo de progresión, se incluyeron en el grupo de peor pronóstico, si bien para ello, asociamos otros factores independientes que influyen en la evolución del propio tumor, en nuestro caso la recurrencia precoz previa y la multiplicidad, implicadas en una mayor frecuencia de progresión y recidiva, respectivamente. De esta manera, identificamos al grupo de casos con peor pronóstico (que denominaremos de alto riesgo), representados por las neoplasias de grado 3 , presencia de Tis y de grado 2 múltiples o que hubieran recurrido previamente en menos de 6 meses. En el estudio del tiempo hasta la progresión observamos claramente diferencias significativas entre este grupo con otros dos: por un lado el de los tumores de grado 1 (únicos o múltiples) y, por otro lado, el de los de grado 2 no múltiples, primarios o recurrentes en más de 6 meses (Fig. 1).

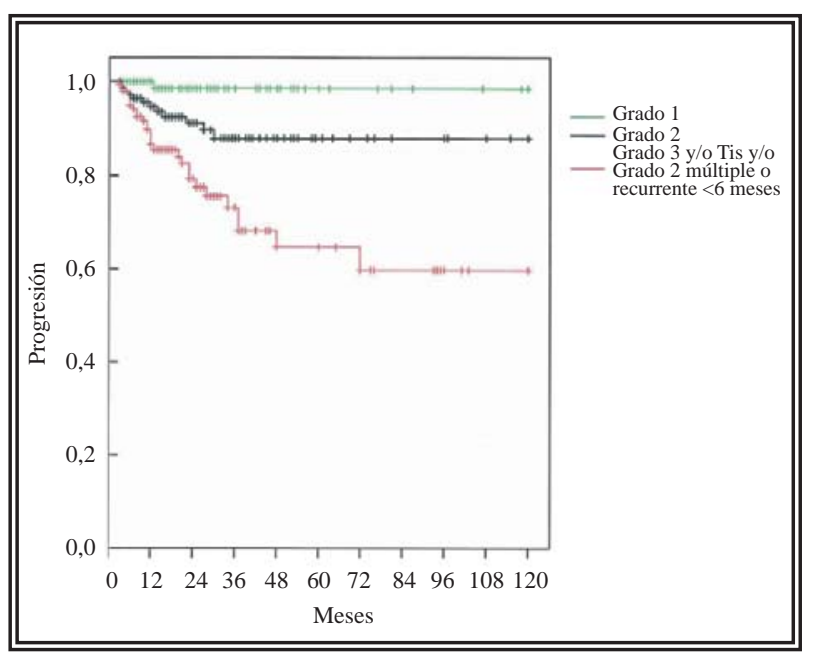

FIGURA 1. Tiempo hasta la progresión según el grado, teniendo en cuenta que el grupo de peor pronóstico incluye los tumores grado 3, tumores con Tis aislado o asociado a una neoplasia exofitica y tumores grado 2 múltiples o recurrentes en menos de 6 meses. Existieron diferencias significativas en el estudio log-rank $(p<0,001)$.

Para establecer el grupo de mejor pronóstico (bajo riesgo), analizamos dentro de los tumores de grado 1 aquellos en los que no existía progresión. Esto se producía en tumores de grado 1 únicos, pues en el tiempo estudiado en nuestra serie no progresaron nunca. En un intento de encontrar un grupo dentro de los de grado 1 únicos con menor tendencia a la recurrencia examinamos diversos factores asociados (tamaño, localización, técnica y estadio), encontrando que en los tumores Ta el tiempo hasta la recurrencia se estabilizaba después del $1^{\circ}$ año tras la RTU. Cuando estratificamos según el $2^{\circ}$ factor implicado en la progresión, es decir, el tratamiento endovesical postRTU, observamos que en los tumores TaG1 únicos, el empleo de terapia endovesical fue 
poco frecuente $\mathrm{y}$, en los casos en los que se empleó, fue fundamentalmente a base de quimioterapia. Al estratificar los casos según el tratamiento empleado observamos que los pacientes que recibieron terapia endovesical no recidivaron, consistiendo en un ciclo de inducción (6 semanas) con quimioterapia con Mitomicina $\mathrm{C}$ en todos los pacientes con neoplasias TaG1 únicas.

En definitiva, por un lado habíamos identificado un grupo amplio de alto riesgo con el que se dejaba escapar el menor número posible de pacientes de mal pronóstico de recurrencia y/o progresión. Por otro lado, se encontró un grupo pequeño en el que estábamos seguros de que no se iba a producir progresión y en el que la recurrencia era mínima. El resto de pacientes constituirían el grupo de pronóstico intermedio.

De esta manera, cuando volvimos a analizar los grupos según el riesgo asociado de recurrencia o progresión, encontramos que existían diferencias estadísticas en el tiempo libre de enfermedad (hasta recurrencia o progresión), con una separación significativa del grupo de bajo riesgo respecto al de medio y alto riesgo que no se diferencian prácticamente (Fig. 2).

Esto parece debido a la influencia de la terapia endovesical, por lo que se analizó el tiempo libre en los casos que no habían recibido este tratamiento. En estos casos se apreciaron diferencias

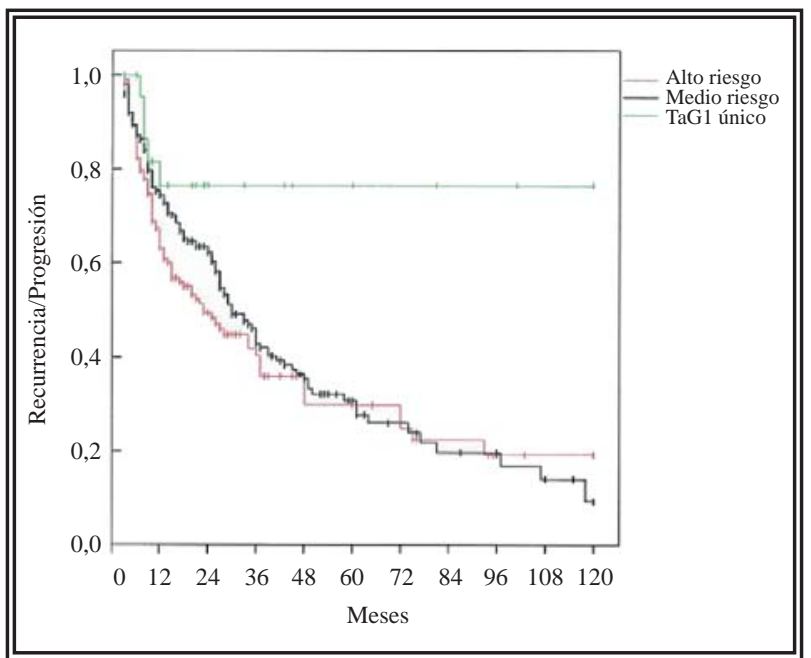

FIGURA 2. Tiempo libre de enfermedad según el grupo de riesgo. Existieron diferencias significativas en la prueba de log-rank (p:0,0083), sin embargo, no existieron entre los grupos de medio $y$ alto riesgo, probablemente debido a la influencia de la terapia endovesical. significativas en el tiempo libre de enfermedad entre los 3 grupos hasta el $5^{\circ}$ año de seguimiento, momento en el que las curvas de los casos de medio y alto riesgo vuelven a converger. Este hallazgo también puede verse desde el inicio cuando sólo se estudiaron los pacientes que habían recibido terapia endovesical postRTU, en los que se evidenció, como dijimos antes, una ausencia de recurrencia en los casos de bajo riesgo (Fig. 3).

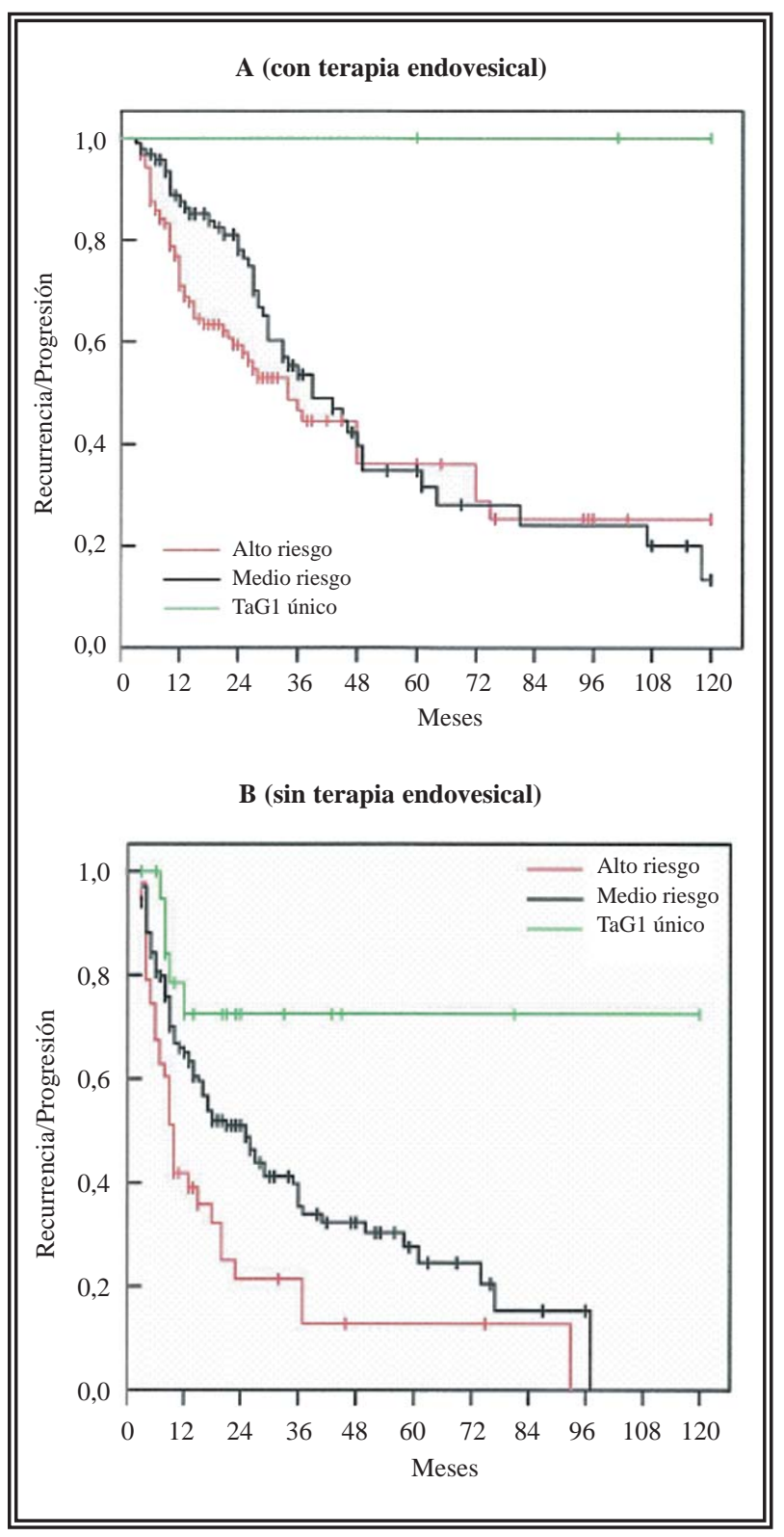

FIGURA 3. Tiempo libre de enfermedad según el grupo de riesgo, estratificando según recibieran $(A)$ o no terapia endovesical (B). 
Por ello, finalmente, creemos que los grupos podrían quedar conformados así (Tabla 2):

- Grupo de buen pronóstico (bajo riesgo): Tumores TaG1 únicos. No existió progresión y el tiempo de recurrencia fue el más largo, con estabilización de la recurrencias tras 1 año.

- Grupo de mal pronóstico: Tumores de alto grado (G3), Tis aislado o asociado y tumores de grado 2 múltiples o recurrentes que hubieran recidivado en menos de 6 meses. No pudimos diferenciar las neoplasias de grado 2 según el estadio (TaG2 vs T1G2), debido a que la mayoría de estos pacientes eran T1G2 (sólo hubo 3 tumores TaG2, que podrian incluirse en alto o medio riesgo).

La mediana del tiempo libre se reduce a 10 meses (8-12 meses; IC del 95\%) en pacientes que no fueron tratados con terapia endovesical, generalmente BCG $81 \mathrm{mg}$ con mantenimiento y se amplia a 25 meses (17-33 meses; IC del 95\%) en los que la recibieron.

La terapia endovesical parece enlentecer la aparición de progresiones, aunque las tasas son similares a largo plazo, con 2 años de diferencia entre aquellos tratados y los que no lo fueron.

- Grupo de pronóstico intermedio: Tumores TaG1 múltiples, así como neoplasias Ta-1G2 y T1G1 no múltiples (menos de 3 tumores). Su tendencia a la recidiva a largo plazo es tan alta como los de alto riesgo pero el riesgo de progresión es mucho menor. El descenso más pronunciado en el tiempo libre de enfermedad se produce en el primer año, con una disminución más lenta que en los tumores de peor pronóstico. La mediana de tiempo libre de enfermedad fue de 30 meses (indice de confianza del 95\% de 25-35). Esta mediana del tiempo libre se reduce a 25 meses

Tabla 2

Grupos de riesgo

\begin{tabular}{ll}
\hline $\begin{array}{l}\text { Grupo de mal pronóstico } \\
\text { (alto riesgo) }\end{array}$ & - G3 \\
& - Tis aislado o asociado \\
& - G2 múltiples o recurrentes \\
& que hubieran recidivado \\
& en menos de 6 meses. \\
& \\
$\begin{array}{l}\text { Grupo de pronóstico } \\
\text { intermedio (medio riesgo) }\end{array}$ & - TaG1 múltiples, \\
& $\begin{array}{l}\text { Ta-1G2 y T1G1 no } \\
\text { múltiples (1 o } 2 \text { tumores) }\end{array}$
\end{tabular}

Grupo de buen pronóstico

(bajo riesgo)

- TaG1 únicos
(17-33 meses; IC del 95\%) en pacientes que no fueron tratados con terapia endovesical y se amplía a 39 meses (26-52 meses; IC del 95\%) en los que la recibieron. No obstante, como se ha dicho, a largo plazo las tasas de recurrencia fueron similares a las de tumores de alto riesgo. Además, la progresión con invasión del detrusor también se produce en los 2 primeros años, estabilizándose a partir del $3^{\mathrm{er}}$ año.

\section{DISCUSIÓN}

Se han realizado varios estudios intentado clasificar los carcinomas superficiales de vejiga en grupos de riesgo observando los factores tradicionales que influyen en la evolución de esta neoplasia $^{4-7,12}$. Entre ellos, destacaremos por su repercusión los de Millán Rodríguez ${ }^{8,13}$ y los de la EAU (European Association of Urology) ${ }^{9,10}$, constituyendo estos últimos la base de las actuales líneas guía de tratamiento y seguimiento del carcinoma vesical superficial. Así, se considera que los tumores únicos Ta G1 menores de $3 \mathrm{~cm}$, son tumores de buen pronóstico, con bajas tasas de recidiva y muy escasa posibilidad de progresión, mientras que los T1, G2-3, Tis deben ser considerados como de alto riesgo, no sólo para recurrencia, sino también para progresión, quedando el resto de tumores en un grupo de riesgo intermedio ${ }^{14}$.

Pagano, en el año 1987 ya publicaba una revisión sobre factores pronósticos en el cáncer de vejiga superficial. Para este autor, el $83 \%$ de tasa de recurrencia que encontraba en su serie estaba influenciada por el número y grado del tumor y por la presencia de displasia. Asimismo, la progresión en el $20 \%$ de los pacientes estaría relacionada con la invasión de la lámina propia, presencia de alto grado, el tiempo libre de enfermedad y la presencia o no de Tis ${ }^{4}$. Estos hallazgos sugieren al autor la necesidad de agrupar los distintos tumores en torno a estas características y plantear así distintos esquemas de seguimiento y tratamiento. En el mismo año, Takashi, clasifica a los pacientes en función de 5 parámetros (T (estadio tumoral), tamaño, síntomas irritativos, edad y grado) sobre los cuales recomienda planificar el tratamiento más adecuado ${ }^{15}$. Llama la atención que para este autor el grado tumoral presente un riesgo relativo de 2,1 resultando ser el menor de todos los parámetros evaluados. 
Desde entonces se han realizado numerosos trabajos para intentar conseguir reunir en grupos de riesgo, a los pacientes con tumor vesical superficial $^{8-10,16-18}$. Su interés radica en poder catalogar a los pacientes, en función de las características del tumor que padecen, en grupos en función a la probabilidad de recurrencia y progresión y así poder optimizar recursos y sobre todo poder ajustar las pautas de tratamiento más adecuadas a cada grupo.

De lo anteriormente comentado, nosotros consideramos tres grupos de riesgo. Para configurarlos nos hemos basado en al análisis estadístico según la odds ratio. Así, por ejemplo, obtenemos con una OR de 2,160 que la multiplicidad ( $>3$ tumores) es un factor de riesgo para recurrencia, asimismo para Oosterlink, la multifocalidad es el factor con mayor peso en la recurrencia del tumor ${ }^{10}$. Objetivamos que el grado no era un factor pronóstico en la recurrencia del tumor, sin embargo analizando la progresión, el grado 3 (respecto al 1) es el que presenta una OR mayor $(20,470)$, similar al publicado por el estudio de Millán et al., donde alcanza una OR de 19,9 para progresión $^{13}$, le siguen con OR menores para riesgo de progresión el grado 2 , la recurrencia en los primeros 6 meses y el empleo de terapia de inducción frente a mantenimiento.

Los grupos finalmente quedaron así configurados:

a) Grupo de mal pronóstico (alto riesgo). Tumores de alto grado (G3), Tis aislado o asociado y tumores de grado 2 múltiples o recurrentes que hubieran recidivado en menos de 6 meses. Coincidimos con el trabajo publicado por MillánRodríguez ${ }^{8}$ excepto en el último factor, que es la recurrencia en menos de 6 meses, no contemplado en ese estudio. Otro autor, Parmar, si incluye en cambio la cistoscopia positiva a los tres meses como factor de riesgo intermedio y alto, aunque sólo para recurrencia ya que no evalúa progresión y mortalidad ${ }^{11}$. Por otra parte la Asociación Europea de Urología (EAU) incluye a los tumores de alta recurrencia dentro del grupo de alto riesgo para recidiva y progresión lo que coincide más con nuestros resultados ${ }^{9,10}$.

En este grupo encontramos claras diferencias en cuanto a tiempo libre de enfermedad y mayor tiempo hasta la progresión utilizando BCG en régimen de mantenimiento, por lo tanto, es lo que recomendamos. Se trata de pacientes que hay que vigilar porque tienen riesgo alto de recurrencia y también pueden progresar.

b) Grupo de pronóstico intermedio. En este grupo se incluyen tumores TaG1 múltiples, así como neoplasias Ta-1G2 y T1G1 no múltiples (menos de 3 tumores). Coincidimos de nuevo con el trabajo de Millán-Rodríguez ${ }^{8}$ en cuanto a considerar los TaG2 únicos o múltiples en este grupo. Respecto al estadio(T), en el estudio de Millán no se considera como un factor pronóstico al igual que en el realizado por Kurth en $1995^{19}$. Otros autores como Herr, sin embargo, consideraban que el estadio tumoral es el único factor pronóstico de progresión al diagnóstico ${ }^{20}$. Para nosotros, los tumores $\mathrm{T} 1 \mathrm{G} 1$ no múltiples se deben incluir en este grupo, porque su tendencia a la recidiva a largo plazo es tan alta como la de los de alto riesgo aunque, es cierto que el riesgo de progresión es mucho menor. La Asociación Europea de Urología los incluye igualmente en el grupo de riesgo intermedio, coincidiendo plenamente con nuestra clasificación ${ }^{9,10}$.

Objetivamos que en este grupo el empleo de quimioterapia o BCG aumentará el tiempo libre de enfermedad global, siendo preferible el mantenimiento y la BCG para disminuir las recurrencias a largo plazo y para evitar la progresión de estadio. Fujii et al. demuestran que el seguimiento de las neoplasias superficiales de vejiga debe ser prolongado, debido a la aparición de recidivas y progresiones tardias, incluso en estos pacientes ${ }^{21}$.

c) Grupo de bajo riesgo. Corresponde a tumores TaG1 únicos, en los que no objetivamos progresión y los tiempos de recurrencia son más largos, estabilizándose tras 1 año de seguimiento donde a partir de entonces no aparecen recidivas en nuestra serie. En algunos casos se podrían emplear ciclos cortos de inducción, consiguiendo evitar las recidivas, aunque la simple vigilancia probablemente sería suficiente ${ }^{22}$.

Otros autores, como Millán-Rodríguez, incluyen en este grupo los $\mathrm{T} 1 \mathrm{G} 1$ únicos, aunque paradójicamente como hemos dicho este autor no tiene en cuenta como factor pronóstico el estadio tumoral cuando realiza el análisis multivariante para recurrencia, progresión y supervivencia libre de enfermedad ${ }^{8,13}$. 
Queremos destacar en nuestro estudio que encontramos diferencias estadísticas al hablar de tiempo libre de enfermedad (tanto hasta la recurrencia como hasta progresión), entre el grupo de bajo riesgo respecto al de medio y alto riesgo, con un mayor tiempo libre de enfermedad del primero. Por otra parte, entre estos dos últimos grupos no encontramos diferencias estadísticamente significativas en la curva de supervivencia para el tiempo libre de enfermedad hasta la recurrencia superficial, aunque sí en cuanto a progresión. Esto difiere del estudio realizado por Millán-Rodríguez ${ }^{8}$, donde demuestran diferencias significativas tanto para recurrencia $(\mathrm{p}<0,05)$ como para progresión $y$ mortalidad, comparando los tres grupos. Esta diferencia en cuanto a la recurrencia en nuestro caso, probablemente se deba a la influencia del tratamiento, al no ser un estudio randomizado, puesto que cuando analizamos este tiempo en los casos que no habian recibido este tratamiento, si apreciamos diferencias significativas en el tiempo libre de enfermedad entre los 3 grupos hasta el 5o año de seguimiento, a partir del cual las curvas de supervivencia vuelven a ser superponibles (Fig. 3).

Algunos estudios han utilizado modelos matemáticos ${ }^{23,24}$ con buenos resultados aunque como hemos comentado, con tiempos de seguimiento relativamente cortos, otros llegan a resultar quizás demasiado complejos para el uso clínico en la practica diaria como por ejemplo el de Takashi que contempla 6 grupos de riesgo ${ }^{15}$.

Nuestro estudio aporta un alto número de pacientes, similar al de otros estudios de factores pronósticos publicados en la bibliografia internacional y con un largo seguimiento. Además, se trata de una serie homogénea de un solo centro y, aunque no es randomizado, los criterios de tratamiento han sido bastante uniformes para todos los pacientes. Por otra parte, se han estudiado de forma multivariante más parámetros que en la mayoría de los estudios previos. Además, valoramos factores de riesgo tanto para recurrencia como para progresión, así como curvas de supervivencia que otros autores, como Parmar ${ }^{11}$, no han valorado (sólo analiza factores pronósticos en cuanto a recurrencia). Kurth et al. proponen un esquema de tratamiento basado en la tasa de recurrencia previa, el tamaño, el grado y el tiempo hasta la primera recurrencia, variables que, en su estudio multi- variante demuestran ser las variables pronósticas independientes para recurrencia, progresión y supervivencia. Posteriormente, establecen una clasificación en 3 grupos de riesgo $y$, aunque, se demuestran diferencias significativas al examinar progresión y mortalidad en el análisis estadístico comparativo entre los grupos de riesgo, no realiza dicho análisis para la recurrencia, por lo que esta clasificación queda parcialmente limitada ${ }^{25}$.

\section{CONCLUSIÓN}

Establecemos así grupos de riesgo en función de factores pronósticos para intentar establecer un mejor control de los pacientes, en cuanto a seguimiento y tratamiento:

1. Grupo de mal pronóstico: Tumores de alto grado (G3), Tis aislado o asociado y tumores de grado 2 múltiples o recurrentes que hubieran recidivado en menos de 6 meses.

Conlleva un alto riesgo de recidiva y progresión. El tratamiento más efectivo en este grupo resultó la BCG con mantenimiento, a pesar del tratamiento, las tasas de recidiva superficial fueron similares a largo plazo exigiendo, por ello, un seguimiento prolongado, más intenso en los 2 primeros años tras la RTU vesical, en los que se produce la mitad de las progresiones.

2. Grupo de pronóstico intermedio (medio riesgo): Tumores TaG1 múltiples, así como neoplasias Ta-1G2 y T1G1 no múltiples (1 ó 2 tumores).

Las tasas de recidiva superficial a largo plazo fueron tan altas como las de alto riesgo, requiriéndose un seguimiento largo.

El riesgo de progresión fue mucho menor que en el grupo 1.

Resultó efectivo el empleo de quimioterapia o BCG siendo preferible el mantenimiento y la BCG para disminuir las recurrencias a largo plazo y evitar la progresión de estadio.

3. Grupo de buen pronóstico (bajo riesgo). Tumores TaG1 únicos.

No existió progresión y el tiempo hasta la recidiva fue el más largo, con estabilización de la tasa de recurrencias tras 1 año.

En los tumores TaG1 únicos, el empleo de terapia endovesical fue poco frecuente y se basó en un ciclo de inducción (6 semanas) con quimioterapia. Los pacientes que recibieron terapia endovesical con inducción no sufrieron recidivas. 


\section{REFERENCIAS}

1. Ruiz Cerdá JL, Alfonso Gil R, Domenech Ferrando E, Pascual Bueno J, Vera Donoso CD, Martínez Jabaloyas J et al. Evolución temporal (1960-1990) de la mortalidad y la razón de masculinidad en España del cáncer vesical. Actas Urol Esp. 1995;19(3):196-202.

2. Messing EM. Tumores uroteliales del tracto urinario. Campbell Urologia. Walsh PC, Retik AB, Vaughan ED, Wein AJ. 8 Ed. Editorial Médica Panamericana. 2004;4(76):2980-3043.

3. Heney NM, Ahmed S, Flanagan MJ, Frable W, Corder MP, Hafermann MD et al. Superficial bladder cancer: progression and recurrence. J Urol. 1983;130(6):1083-1086.

4. Pagano F, Garbeglio A, Milani C, Bassi P, Pegoraro V. Prognosis of bladder cancer. I. Risk factors in superficial transitional cell carcinoma. Eur Urol. 1987;13(3):145-149.

5. Chen SS, Chen KK, Lin AT, Chang YH, Wu HH, Hsu TH et al. The significance of tumor grade in predicting disease progression in stage Ta transitional cell carcinoma of the urinary bladder. Br J Urol. 1996;78(2):209-212.

6. Sánchez de la Muela P, Rosell D, Aguera L, De Castro F, Isa W, Robles JE et al. Multivariate analisis of progression in superficial bladder cancer. Br J Urol. 1993;71(3):284-289.

7. Raitanen MP, Nieminen P, Tammela TL. Impact of tumor grade, stage, number and size, and smoking and sex, on survival patients with transitional cell carcinoma of the bladder. Br J Urol. 1995;76(4):470-474.

8. Millán-Rodríguez F, Chechile-Toniolo G, Salvador-Bayarri J, Palou J, Algaba F, Vicente-Rodríguez J. Primary superficial bladder cancer risk groups according to progression mortality and recurrence. J Urol. 2000;164(3):680-684.

9. Oosterlinck W, Lobel B, Jakse G, Malmström PU, Stöckle M, Sternberg C. Guidelines on bladder cancer. Eur Urol. 2002;41(2):105-112.

10. Oosterlinck W. Guidelines on diagnosis and treatment of superficial bladder cancer. Minerva Urol Nefrol. 2004;56 (1):65-72.

11. Parmar MK, Freedman LS, Hargreave TB, Tolley DA. Prognostic factors for recurrence and followup policies in the treatment of superficial bladder cancer: report from the British medical research council subgroup on superficial bladder cancer (Urological cancer working party). J Urol. 1989; 142(2 Pt1):284-288.

12. Busto Catañón L, Sánchez Merino JM, Picallo Sánchez JA, Gelabert Mas A. Factores pronósticos clínicos en el cáncer superficial vesical. Arch Esp Urol. 2001;54(2):131-138.

13. Millán-Rodríguez F, Chechile-Toniolo G, Salvador-Bayarri J, Palou J, Vicente-Rodríguez J. Multivariate analysis of the prognostic factors of primary superficial bladder cancer. J Urol. 2000;163(1):73-78.

14. Bohle A, Jocham D, Bock PR. Intravesical bacillus Calmette-Guerin versus mitomycin $\mathrm{C}$ for superficial bladder cancer: a formal meta-analysis of comparative studies on recurrence and toxicity. J Urol. 2003;169(1):90-95.
15. Takashi M, Murase T, Mizuno S, Hamajima N, Ohno Y. Multivariate evaluation of prognostic determinants in bladder cancer patients. Urol Int. 1987;42(5):368-374.

16. Ali-El-Dein B, Sarhan O; Hinev A, Ibrahiem el-HI, Nabeeh A, Ghoneim MA. Superficial bladder tumours: analysis of prognostic factors and construction of a predictive index. BJU Int. 2003;92(4):393-399.

17. Santos L, Amaro T, Costa C, Pereira S, Bento MJ, Lopes P, et al. Ki-67 index enhances the prognostic accuracy of the urothelial superficial bladder carcinoma risk group classification. Int J Cancer. 2003;105(2):267-272.

18. Vicente R. Propuesta de seguimiento en Fundación Puigvert. J. Vicente R, G. Chéchile, J Salvador. Tumores vesicales superficiales Edit. Acción médica SA., 2000;215223.

19. Kurth KH, Bouffioux C, Sylvester R, Van der Meijden APM, Oosterlinck W, Brausi M. Treatment of superficial bladder tumors: achievements and needs. The EORTC Genitourinary. Group. Eur Urol. 2000;37(Suppl 3):1-9.

20. Herr HW, Badalament RA, Amato DA, Laudone VP, Fair WR, Whitmore WF Jr. Superficial bladder cancer treated with bacillus Calmette-Guerin: a multivariate analysis of factors affecting tumor progression. J Urol. 1989;141(1): 22-29.

21. Fujii Y, Fukui I, Kihara K, Tsujii T, Kageyama Y, Oshima H. Late recurrente and progresión alter a long tumor-free period in prymary Ta and T1 bladder cancer. Eur Urol. 1999;36(4):309-313.

22. Andius P, Holmang S. Bacillus Calmette-Guerin therapy in stage Ta/T1 bladder cancer: prognostic factors for time to recurrence and progression. BJU Int. 2004;93(7):980-984.

23. Jiménez Cruz JF, Llopis Minués B, Vera Donoso CD. Cáncer vesical superficial primario. Factores pronósticos para recurrencia. Arch Esp Urol. 1990;43(Suppl 2):139147.

24. Allard P, Bernard P, Fradet Y, Tetu B. The early clinical course of primary $\mathrm{Ta}$ and $\mathrm{T} 1$ bladder cancer: a proposed prognostic index. Br J Urol. 1998;81(5):692-698.

25. Kurth KH, Denis L, Bouffioux C, Sylvester R, Debruyne FM, Pavone-Macaluso M et al. Factors affecting recurrence and progression in superficial bladder tumors. Eur J Cancer. 1995;31A(11):1840-1846.

Dr. J. García Rodríguez

E-mail: jgrmed@hotmail.com

(Trabajo recibido el 9 de marzo 2006) 